\title{
Editorial
}

\section{How much bond strength do we need?}

The retention of restorative materials was always a challenge to the dental professionals throughout history. Before the development of bonding to tooth substrate, mechanical retention shapes, such as undercuts in cavity preparation, were widely used, resulting in additional removal of sound tooth structure. The beginning of adhesive dentistry, with the classical study of Prof. Buonocore, in 1955, put into our minds the importance of bond strength. He tested the resistance to removal by 'thumbnail pressure' of acrylic drops placed on treated and untreated enamel surfaces of extracted teeth using phosphoric acid. The success obtained on enamel was not the same as on dentin, because of many differences between those substrates. Many efforts were made by a lot of researches to obtain a reliable bonding system to dentin. During this evolution, the bond strength measuring methods were considered as essential tools to predict the efficiency of an adhesive material, and different testing methods were also developed, such as shear and tensile tests, which were improved and resulted in the microtensile and microshear tests used nowadays.

The first dentin bonding materials obtained very low bond strength values, such as 5-7 MPa, due to their interaction with the smear layer, instead of the underlined dentin. The clinical performance of those materials was very poor and interfacial staining and lack of retention was a real problem. With the introduction of dentin conditioning by Prof. Fuzayama and the development of the hybrid layer concept, resulting from the remarkable work of Prof. Nakabayashi, the studies started to show higher bond strength values, never seen before. The great success of this class of new materials represented a huge change in every day dental practice, opening the doors to the development of a new conservative dentistry, in which the preservation of tooth structure is the main objective. With this advance, the cavity preparations for direct composite restorations were reduced to a simply removal of carious enamel and infected dentin, eliminating the necessity of a 'stereotyped cavity design.'

After this point, the industries started a kind of 'bond strength competition.' Each one claimed that its product resulted in higher values in relation to the others, which would represent a better clinical performance. As an advertising strategy, the bond strength values could be seen on labels of some materials and on technical profiles. However, when scientific literature is analyzed, one can find many investigations comparing the bond strength among different brands of bond systems. In most of them, the difference was relatively very low, such as 20 in relation to 25 or $30 \mathrm{MPa}$. What does this difference mean? If the results of clinical trials were analyzed, sometimes similar results were obtained after 2 to 3 years with those same materials. Therefore, the researchers started to raise a question. What is the clinical relevance of such bond strength studies?

At the last Academy of Dental Materials meeting held in October 2011, the main question discussed was exactly that. What is the clinical relevance of in vitro tests? Are they reliable to predict the clinical performance of bonding systems and restorative materials? There are many evidences that unfavorable results of bond strength tests or microleakage studies do not always mean a bad clinical behavior. The opposite is also true. The materials that show the highest bond strength values are not necessarily better clinically than others with lower values. One of the conclusions of the meeting was that long-term clinical trials, such as 10 or more years, are the most important studies to prove which material is really the best. However, such kinds of studies are expensive and time-consuming and, sometimes, at the end the materials tested are no longer available at the market. Therefore, probably a better in vitro model must be created to predict at the laboratory, what is the better material to use clinically.

Probably, there is a minimum level necessary in relation to bond strength values. If you use a bonding system which gives you just $2 \mathrm{MPa}$ of bond strength, you will for sure experience a bad clinical performance at the margins without enamel and probably loss of retention in many restorations. However, when you reach a certain level of bond strength, actually not yet determined, the clinical behavior of that restoration will be similar to the others with higher MPa values, at least in short time periods (1-2 years). Of course, there are many other variables to obtain a good quality restoration, such as the sealing ability of the adhesive system, the longevity of bonding, the selection of a good restorative material as well as the skills of the operator who is performing the job, maybe with a higher impact on final clinical behavior. The main conclusion that the clinicians must have in their minds is that the results of in vitro studies are important for the manufacturers to create a good material, but the clinical studies and their own experiences with that product must be the guide to select what material they will use in their patients’ mouth.

Carlos Rocha Gomes Torres Assistant Professor Department of Restorative Dentistry São Paulo State University - UNESP São José dos Campos, São Paulo, Brazil 